\section{B-cell function modulation in multiple sclerosis: a new therapeutic paradigm}

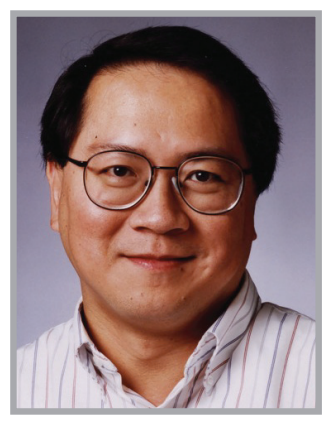

Andrew Chan': Andrew Chan MD, PhD obtained his bachelor's and master's degrees in chemistry from Northwestern University in Evanston (IL, USA) and his medical degree and doctorate in cellular and developmental biology from the Washington University School of Medicine in St Louis (MO, USA). He completed an internship and residency in internal medicine at Barnes Hospital at Washington University School of Medicine before becoming a postdoctoral clinical and research fellow at the University of California, San Francisco (CA, USA). Dr Chan is Senior Vice President of Research Biology of Genentech Inc (CA, USA). Dr Chan's current focus is to ensure that we are at the forefront of discovering new biologies, dissecting the heterogeneous causes of human disease, and developing new therapies to attack the dominant pathogenic pathways of disease. Dr Chan's laboratory is currently working on defining the role of immune cells in autoimmune disease, as well as understanding the mechanisms of action of anti-CD20 and other B-cell modulatory therapies. His research interests include basic discovery in understanding the underlying pathogenesis of human autoimmune and inflammatory disorders. He is also a frequent lecturer and has authored or coauthored more than 100 scientific articles. Prior to joining Genentech in 2001, Dr Chan was an associate professor in the Division of Rheumatology and the Departments of Medicine, Pathology and Immunology at Washington University School of Medicine, where he was also a Howard Hughes Medical Institute associate investigator. Dr Chan is a member of the American Association of Immunologists, the American Society for Clinical Investigation and the American Association of Physicians.

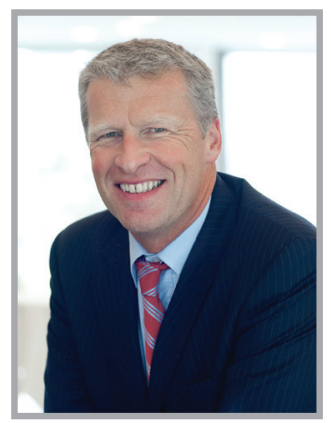

Albert Jan Schutte 2 : In 2008 Albert joined F HoffmannLa Roche Ltd in Basel (Switzerland) from Schering-Plough/ Organon (NJ, USA) to take over the leadership of the Ocrelizumab Multiple Sclerosis Life Cycle team. Since February 2013, he is the Global Medical Director for Neuroscience and Cardiometabolism. Albert qualified as an MD at the Free University of Amsterdam (The Netherlands) and worked several years in Internal Medicine in Amsterdam. He started his career in pharma as a Medical Advisor CNS with Solvay Pharma (Weesp, The Netherlands). He then joined Solvay Pharma International where he, as Medical Manager, was responsible for the Middle East and Asian Pacific Region. In 1998, he joined Organon International (NJ, USA) where he worked almost exclusively in neuroscience (depression, bipolar disorder and schizophrenia). In 2002, he joined the US Headquarters, where he was Executive Director Neuroscience, acting both as Global Marketing Director and Global Medical Science Lead.

'Research Biology, Genentech, Inc., South San Francisco, CA, USA; chan.andy-research@gene.com

${ }^{2}$ F Hoffmann-La Roche Ltd, Grenzacherstrasse 183, Building 74/3E.Z02.09, 4070 Basel, Switzerland; albert_jan.schutte@roche.com
Niêteroologr

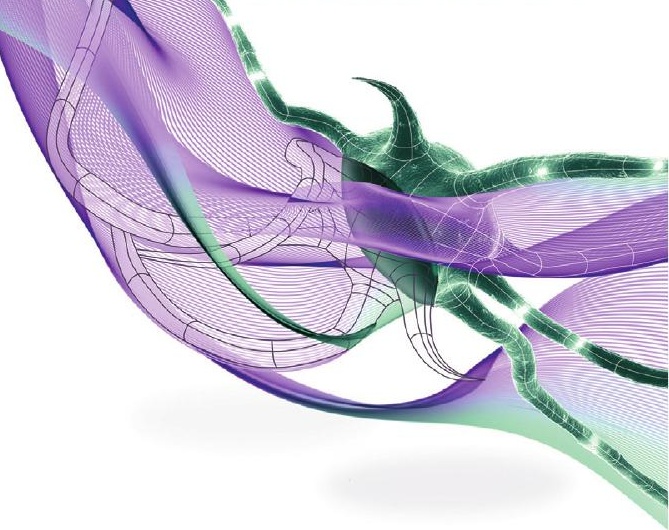


Q Can you explain a little about your roles at Roche?

Dr Chan - I am the Senior Vice President of the research biology organization of Genentech in San Francisco (CA, USA). I head the biology areas in Genentech San Francisco. These include the areas of oncology, immunology, infectious diseases, neuroscience and ocular diseases.

Dr Schutte - I am global medical director of Medical Affairs in neuroscience and cardiometabolism in Roche and based in Basel. I head a team of international medical leaders that, amongst others, include teams working on latestage development programs in schizophrenia, multiple sclerosis (MS) and Alzheimer's disease.

Q Can you describe the rationale behind targeting B cells for the treatment of MS?

Dr Chan - From our experience, over the past decade or so, we have known that $\mathrm{B}$ cells have significantly important roles in a number of autoimmune disorders. It has been appreciated that $\mathrm{B}$ cells are actually central in guiding the activation of a number of immune cells in the body, including $\mathrm{T}$ cells, dendritic cells and others. B cells actually play a central role in a number of autoimmune diseases, including MS.

There are a lot of additional preclinical data trying to understand precisely how the B cells guide the entire immune system for MS. As I mentioned, B cells actually control the activation of $\mathrm{T}$ cells, so in a number of mouse models, where one gets rid of the B cells, the T cells actually become quiet, no longer able to go and destroy other cell types and tissues. In fact, in MS, B cells had been suggested to guide a particular subset of T cells called Th17 cells, which have been demonstrated to have a role in promoting MS disease.

\section{Q Roche is currently developing the antibody ocrelizumab for the treatment of MS. Could you describe its history and mechanisms of action?}

Dr Chan - Genentech/Roche developed the first anti-CD20 antibody, rituximab, which was approved in 1997 for the treatment of nonHodgkin's lymphoma [1]. We also have a history elucidating the potential benefits of B-cell therapy for the treatment of autoimmune diseases, culminating in the 2006 approval of rituximab for the treatment of rheumatoid arthritis. Then, later in 2006, we made the discovery that B cells play an important role in MS with the results of HERMES, a Phase II study of rituximab in relapsing remitting $\mathrm{MS}$ [2]. This was reported in 2008 in the New England Journal of Medicine. At that time, based on our knowledge of monoclonal antibodies in autoimmune disease, the use of chimeric antibodies (rituximab is a chimeric antibody) was not an ideal choice as a treatment option for chronic conditions. We therefore generated a humanized $\mathrm{mAb}$, called ocrelizumab, for the treatment of MS because it's much more of a chronic disease than some of the B-cell malignant diseases. This humanized antibody has human components in well over $95-96 \%$ of the amino acids. It has a better immunogenicity profile. When we use chimeric antibodies such as rituximab in the chronic setting, patients can develop their own immune response to the drug (antidrug antibodies). In the long term, the development of these antidrug antibodies is not good for patients in the chronic setting because they clear and get rid of the drug that is being given. So it was with that intent that we began these studies with ocrelizumab in MS.

Q Can you explain a little about the trials that have been performed so far?

Dr Schutte - The HERMES study was a proofof-concept study of rituximab in patients with relapsing-remitting MS, providing the first clinical data that supported the theory behind $\mathrm{B}$ cells in MS. In HERMES, we saw a surprisingly high efficacy, as measured by a reduction in gadolinium-enhancing lesions, a marker of inflammatory activity in the brain that can be quantified on MRI scans. In addition, there was also a reduction in annualized relapse rate, a clinical measure of disease activity. Nobody expected the effect to be so profound because, until then, the disease was considered to be primarily a T-cell-mediated disease.

The ocrelizumab Phase II study consisted of a 24-week primary comparison of ocrelizumab (one treatment course of two infusions) versus placebo and an exploratory comparison with IFN- $\beta$-1a in patients with relapsing-remitting MS [3]. Thereafter, all patients received openlabel ocrelizumab for three courses up to 2 years and were then followed for safety for an additional year. The results from this study supported what we saw with rituximab in the proof-of-concept study. There was a significant decrease in gadolinium-enhancing lesions - lesions were reduced by $89 \%$ versus 
placebo. For the annualized relapse rate, we realized there was a reduction of $80 \%$ versus placebo and $66 \%$ versus interferon. Therefore the results of the ocrelizumab confirmed our assumption that B-cell therapy could potentially be a new treatment strategy in MS.

\section{Q Can you explain a little about the Phase III program?}

Dr Schutte - The ocrelizumab Phase III clinical programme (ORCHESTRA) that has completed enrolment consists of two studies in patients with relapsing MS (OPERA I and II) and one study in patients with primary progressive MS (ORATORIO) [101-103]. In the relapsing MS trials we are comparing ocrelizumab with placebo and IFN- $\beta$. The study is ongoing and we expect results in 2015 .

The ORATORIO study in primary progressive MS is based on the OLYMPUS primary progressive MS trial with rituximab. Although the primary end point was not met in OLYMPUS, a prespecified subgroup analysis showed that rituximab had a treatment effect in younger patients who had more active disease by the presence of gadolinium-positive lesions. Based on that data, we took ocrelizumab forward in Phase III in primary progressive MS, a patient population for which there is an unmet medical need because there is currently no approved products for the treatment of primary progressive MS.

\section{Q Have the trials so far produced any surprising results or are they as you were hoping and expecting?}

Dr Schutte - The Phase III trials are ongoing and still blinded, so we don't know their outcome yet. But the Phase II results were quite encouraging in terms of the high level of efficacy that was observed [3].

\section{Q Why is targeting B cells with anti-CD20 antibodies such an appealing treatment strategy?}

Dr Chan - There has been a concern with B-cell treatment strategies because there has been a misperception that a consequence of reducing $\mathrm{B}$ cells results in a loss of a significant part of the immune protection. But anti-CD20 antibodies represent a fairly safe approach in this regard. While a subset of the $\mathrm{B}$-cell repertoire is significantly reduced with anti-CD20 therapies, the plasma cells that are the end product of the B cells do not express CD20. This means that the plasma cells that make all of the antibodies that protect against everyday diseases are preserved. Thus these anti-CD20 antibodies selectively remove the fraction of the immune system that is promoting inflammation in MS, but preserve the critical portion that is needed for protection against infection. So far, it appears that the benefit-risk ratio is actually quite favorable for patients with autoimmune diseases such as rheumatoid arthritis and MS.

Q Are there any other treatment avenues that are currently being taken by Roche for the treatment of MS?

Dr Chan - Yes, we have a number of earlier programs that have not yet gone through the proof-of-concept Phase II trials, but we have a significant level of research and early clinical interest in this area that we intend to take into MS-based research; however, right now it is too early to discuss.

\section{Q Are there any other neurodegenerative} diseases for which a similar treatment strategy could be used?

Dr Chan - Without a doubt. In MS there are two major components of the disease; inflammation and neurodegeneration. The hope is that by targeting the inflammatory component we can prevent patients from deteriorating in terms of the neurodegenerative component.

We have other programs that are in Phase II and III that target the amyloid- $\beta$ component of Alzheimer's disease. However, the causes for neurodegeneration in MS are quite distinct from other classic neurodegenerative diseases like Alzheimer's disease, so we have not pursued ocrelizumab in the treatment of these diseases, but Roche/Genentech is quite active in research and development of potential new treatments for neurodegenerative diseases.

Q What further research does Roche plan to conduct in this field?

Dr Schutte - Since the early 20th century Roche had a focus in psychiatry, and have developed and marketed products that include Pantopon, Anexate and Valium. Roche is now re-entering the neuroscience field and focusing on three primary areas - neurodegeneration, psychiatry and neurodevelopmental disorders - specifically those areas where there is still a high, unmet need or where there are no available treatments. One example is ocrelizumab, but also we have 
another Phase III stage product in development called bitopertin, which is a compound that treats the negative symptoms of schizophrenia; there is no approved treatment for that condition at the moment. In addition, there is a Phase III development program for Alzheimer's disease with a drug called gantenerumab. So Roche is really getting back into neuroscience with an innovative pipeline and interesting compounds, with new modes of action.

Dr Chan - Right now, MS is at an extremely exciting time for drug discovery and development. There have been a number of major advances over the past 5 years that make us want to enter the field. These include all the human genomics research that has been done, understanding the genes that increase risk for patients. There are a number of whole-genome sequences identifying the pathways that govern susceptibility. I therefore think the entire area of neurodegeneration, which has always been a black box, is slowly being opened. The application of these and other novel technologies to better understand the risk factors that contribute to and biologies that cause disease will provide a number of opportunities for us and the industry to make substantial advances in the treatment of MS and neurodegenerative diseases.

\section{Disclaimer}

The opinions expressed in this interview are those of the interviewees and do not necessarily reflect the views of Future Medicine Ltd.

Financial \& competing interests disclosure

A Chan is an employee of Genentech, Inc. AJ Schutte is an employee of F Hoffmann-La Roche Ltd. The interviewees have no other relevant affiliations or financial involvement with any organization or entity with a financial interest in or financial conflict with the subject matter or materials discussed in the manuscript apart from those disclosed.

No writing assistance was utilized in the production of this manuscript.

\section{References}

1 Grillo-Lopez AJ, White CA, Varns C et al. Overview of the clinical development of rituximab: first monoclonal antibody approved for treatment of lymphoma. Semin. Oncol. 26, 66-73 (1999).

2 Hauser SL, Waubant E, Arnold DL et al. $\mathrm{B}$-cell depletion with rituximab in relapsingremitting multiple sclerosis. N. Engl. J. Med. 358, 676-688 (2008).
3 Kappos L, Li D, Calabresi PA et al. Ocrelizumab in relapsing-remitting multiple sclerosis: a Phase 2, randomised, placebocontrolled, multicentre trial. Lancet 378(9805), 1779-1787 (2011).

\section{- Websites}

101 A Study of Ocrelizumab in Comparison With Interferon Beta-1a (Rebif) in Patients With Relapsing Multiple Sclerosis. www.clinicaltrial.gov/ct2/show/ NCT01247324

102 A Study of Ocrelizumab in Comparison With Interferon Beta-1a (Rebif) in Patients With Relapsing Multiple Sclerosis. www.clinicaltrial.gov/ct $2 /$ show/ NCT01412333

103 A Study of Ocrelizumab in Patients With Primary Progressive Multiple Sclerosis. www.clinicaltrial.gov/ct2/show/ NCT01194570 